\title{
Prevalence and severity of malnutrition in pediatric neurology outpatients with respect to underlying diagnosis and co- morbid nutrition and feeding related problems
}

\author{
Hande Tekin, Hasan Tekgül, Sanem Yılmaz, Dilek Arslangiray, Hediye Reyhan, \\ Gül Serdaroğlu, Sarenur Gökben \\ Division of Child Neurology, Department of Pediatrics, Ege University Faculty of Medicine, Izmir, Turkey. \\ E-mail: hasan.tekgul@ege.edu.tr \\ Received: 27th February 2018, Accepted: 16th March 2018
}

\begin{abstract}
SUMMARY: Tekin H, Tekgül H, Yılmaz S, Arslangiray D, Reyhan H, Serdaroğlu G, Gökben S. Prevalence and severity of malnutrition in pediatric neurology outpatients with respect to underlying diagnosis and co-morbid nutrition and feeding related problems. Turk J Pediatr 2018; 60: 709-717.
\end{abstract}

This study aimed to determine prevalence and severity of malnutrition with respect to underlying diagnosis and co-morbid nutrition and feeding related problems in pediatric neurology outpatients. A total of 1,057 pediatric neurology outpatients $(7.2 \pm 5.4$ years, $56.9 \%$ males) were included. Data on patient demographics, neurological diagnosis, anthropometrics and Nutritional Questionnaire (NQ) for co-morbid feeding difficulties and nutritional problems were recorded. Epilepsy $\mathbf{( 4 5 . 2 \% )}$ was the most common diagnosis, while prevalence of acute malnutrition was $17.7 \%$. Nutritional support resulted in a significant decrease in the percentage of malnourished patients (from $17.1 \%$ to $6.7 \%, \mathrm{p}<0.001$ ) and significant improvement in weight for height scores (increased to 81.42 $\pm 8.17, \mathrm{p}=0.045$ ). In NQ-10 item assessment, at least one item was positive in $66.0 \%$ (gastrointestinal in $54.3 \%$ ) of acutely malnourished patients, more commonly in severe acute malnutrition. NQ 4item set of "red flags" revealed that prolonged meal time, meal time stressful to child or parent, lack of weight gain not just weight loss and cough during feeding were evident in $45.2 \%, 46.8 \%, 36.7 \%$ and $14.8 \%$ of patients with acute malnutrition, respectively; and more common in patients with severe malnutrition. NQ 4-item set of "red flags" was associated with high sensitivity (95\%) and specificity $(88 \%)$ in detection of malnutrition. In conclusion, our findings in a cohort of pediatric neurology outpatients revealed that $17.1 \%$ of overall patients were acutely malnourished along with higher prevalence of malnutrition in underlying diagnosis of cerebral palsy and higher likelihood of nutritional problems and feeding difficulties in severe malnutrition. Given the association of 6-month nutritional support with improved anthropometrics and decreased percentage of malnourished patients, our findings indicate that increased awareness of nutritional status and nutritional support is essential for the care of neurologically impaired children with potential benefit of identifying early feeding/swallowing related signs of malnutrition.

Key words: pediatric neurology, anthropometrics, malnutrition, nutritional status, questionnaire.

Neurodevelopmental disabilities are frequently associated with factors leading to malnutrition such as decreased food intake, increased nutrient loss and nutrient requirement. ${ }^{1}$ Accordingly, significant proportion children with neurological disabilities are considered to be at high risk of poor nutritional status, particularly those with severe and longer term gross motor impairment and oropharyngeal dysfunction. $^{2-4}$

Assessment of nutritional status and implementation of appropriate nutritional support when necessary are therefore considered essential for the optimal care in 
these patients and become instrumental in improving nutritional status with the help of multidisciplinary feeding programs. ${ }^{2,5}$ However, despite guideline recommendations ${ }^{6}$, nutrition screening has not been widely applied in clinical practice $^{7}$, possibly due to insufficient nutrition knowledge among physicians ${ }^{8}$ as well as lack of a gold standard validated pediatric nutrition screening tool. ${ }^{9,10}$

In fact, children with neurological impairment frequently have feeding difficulties and nutritional problems with close association of growth retardation with inadequate intake as a result of self-feeding impairment and oromotor dysfunction ${ }^{11}$. However, nutritional and feeding problems in children with disabilities are under recognized with advanced technology and progress in terms of ambulation, communication, education, and orthopedic care rather than the nutritional care in the clinical management of these children. ${ }^{11}$

Growth failure and undernutrition are not inevitable and irremediable consequences of neurological disability and are often correctable via inclusion of nutritional assessment and management as part of the routine care. ${ }^{11-13}$

This prospective study was therefore designed to determine prevalence and severity of malnutrition in pediatric neurology outpatients with respect to underlying diagnosis and comorbid nutrition and feeding related problems and to determine the impact of 6-month nutritional support on nutritional status in malnourished patients.

\section{Material and Methods}

\section{Study population}

A total of 1,057 children with neurological disabilities (mean age: $7.2 \pm 5.4$ years; 601 males, 456 females) followed up at a tertiary care pediatric neurology outpatient clinic between June 2015 and December 2015 were included in this cross-sectional prospective study. Patients with diseases that are likely to cause significant changes in body weight including severe heart disease, renal failure, hepatic failure, dehydration and fluid imbalance such as third space shift were excluded from the study.

Written informed consent was obtained from parent/legal guardian of each patient following a detailed explanation of the objectives and protocol of the study which was conducted in accordance with the ethical principles stated in the "Declaration of Helsinki" and approved by the Institutional Ethics Committee (Date of approval: 07/02/2017; Reference number: 17-2/ 48).

\section{Study parameters}

Data on patient demographics, neurological diagnosis, anthropometrics and Nutritional Questionnaire (NQ) for co-morbid feeding difficulties and nutritional problems were recorded and evaluated with respect to presence of malnutrition and nutritional support.

\section{Anthropometric measurements}

Anthropometrics included measurement of weight, height, mid-upper arm circumference (MUAC) and triceps skinfold thickness (TSFT) by the same nurse (DA). Body weight was measured using a digital baby weight scale (Terraillon, France; $10 \mathrm{~g}$ precision) in children aged $\leq 2$ years, while with adult electronic scale (Uwe-PM 150, UK, $100 \mathrm{~g}$ precision) in children aged $>2$ years. Height measurement was performed using a 1-m length measuring tape $(0.1 \mathrm{~cm}$ precision $)$ in children aged $\leq 2$ years and with a wall mounted stature meter $(0.2 \mathrm{~cm}$ precision) in children aged $>2$ years. MUAC was measured from the left upper arm flexed slightly at the elbow, at half distance between the acromion and the olecranon using a plastic measuring tape. TSFT was measured from the left arm, and at half distance between the acromion and the olecranon, using Holtain skin fold caliper (Holtain, Chymycin, UK).

\section{Nutritional Questionnaire (NQ)}

NQ is a 14-item questionnaire for assessment of feeding and nutrition in children with neurological disorders. First set of the questionnaire includes four key questions called "red flags" asked to parents [(1) How long does it take to feed your child? (2) Are meal times stressful to child or parent? (3) Is your child gaining weight adequately? (4) Are there signs of respiratory problems?], as described previously by Arvedson. ${ }^{14}$ The second set includes screening for nutrition related problems based on a list of 10 items 
likely to be encountered in children with neurologic disorders [(1) vomiting, (2) diarrhea, (3) constipation, (4) difficulty in swallowing, (5) abdominal pain, (6) loss of appetite, (7) nausea/regurgitation, (8) oral and dental problems, (9) depression/irritability and (10) economic problems]. Patients were consulted with appropriate specialist depending on the existing problem, including a pediatric gastroenterologist (any of the first seven items), a pediatric dentist (item 8 ), a child psychiatrist (item 9) or a social worker (item 10).

\section{Malnutrition assessment and nutritional support}

Diagnosis of malnutrition was based on anthropometric measurements performed by the same physician (HG) in the first week of neurologic examination. Weight for height (WFH), height for age (HFA) and body mass index values were calculated and TSFT was measured in each patient, and findings were assessed using National Center for Health Statistics (NCHS) charts or standardized data. Using Waterlow Classification system, wasting (the percentage of expected WFH) and the degree of stunting (the percentage of expected HFA) were determined to diagnose acute malnutrition (WFH\% $<90 \%$ and HFA $>95 \%$ ) and chronic malnutrition (WFH\% $90-110 \%$ and HFA <95\%). Cases with acute malnutrition were further categorized in terms of severity based on WFH\% [normal (>90\%), mild $(80-90 \%)$, moderate $(70-80 \%)$, severe $(<70 \%)$ ] and \%HFA [normal $(>95 \%)$, mild (90-95\%), moderate $(85-90 \%)$, severe $(<85 \%)]$ parameters.

Nutritional support was administered in malnourished patients in accordance with the calorie and appropriate feeding route (oral, nasogastric tube or gastrostomy) for 6 months. Anthropometric measurements were repeated at the $3^{\text {rd }}$ month and $6^{\text {th }}$ month of nutritional support and compared with baseline data.

\section{Statistical analysis}

Statistical analysis was made using IBM SPSS Statistics (IBM Corp. Released 2012. IBM SPSS Statistics for Windows, version 21.0, Armonk, NY). Chi-square test was used for the comparison of categorical data, while t-test was used for the analysis of numerical data. The sensitivity, specificity, positive predictive value
(PPV) and negative predictive value (NPV) of "red flags" scores in estimation of malnutrition were calculated. Data were expressed as mean \pm SD (standard deviation, and percent (\%), where appropriate. A p value $<0.05$ was considered statistically significant.

\section{Results}

\section{Baseline characteristics}

Study population was composed of 601 males and 456 females with a mean age of $7.2 \pm 5.4$ years. Epilepsy (45.2\%) and cerebral palsy (CP; $15.8 \%$ ) were the two most common diagnoses, while acute malnutrition was diagnosed in 188 $(17.7 \%)$ cases and chronic malnutrition was present in 117 (11.1\%) cases (Table I).

\section{Malnutrition with respect to underlying diagnosis and nutritional support}

Acute (mild in majority of cases) malnutrition rate was $36.0 \%, 28.4 \%$ and $13.8 \%$ in patients with CP, neuro-metabolic disorders, and epilepsy, respectively (Table II).

Clinical nutrition was administered via diet per se in $60(45.8 \%)$ out of 131 patients with malnutrition, while diet plus enteral supplements was the choice of nutritional support for the remaining malnourished patients.

Table I. Baseline Characteristics of the Study Population $(\mathrm{N}=1057)$.

\begin{tabular}{lc}
\hline Age, years & $7.2 \pm 5.4$ \\
Gender, N (\%) & \\
Female & $456(43.1)$ \\
Male & $601(56.9)$ \\
Neurological disease, N (\%) & \\
$\quad$ Epilepsy & $478(45.2)$ \\
Cerebral palsy & $167(15.8)$ \\
Neuro-muscular disorders & $103(9.8)$ \\
Neuro-metabolic disorders & $95(9.0)$ \\
Neuro-immune disorders & $71(6.7)$ \\
Other & $143(13.5)$ \\
Malnutrition, N (\%) & $305(28.9)$ \\
Acute malnutrition & $188(17.7)$ \\
$\quad$ Mild & $131(12.3)$ \\
$\quad$ Moderate & $44(4.1)$ \\
$\quad$ Severe & $13(1.2)$ \\
Chronic malnutrition & $117(11.1)$ \\
\hline
\end{tabular}




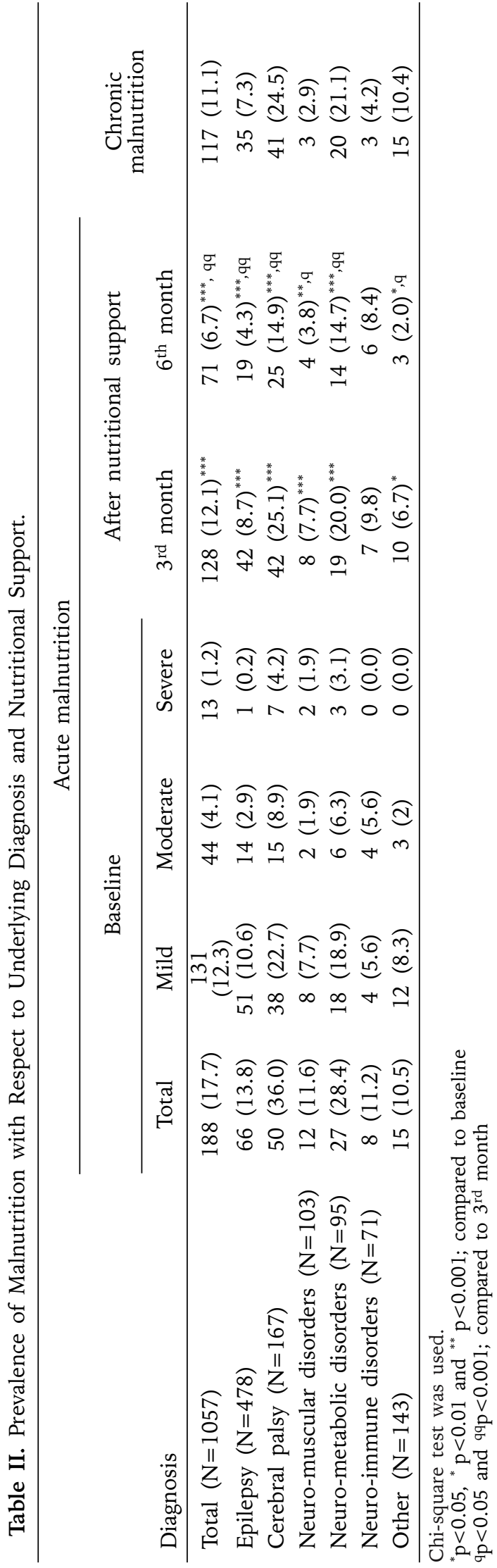

After 3-month and 6-month long nutritional support, a significant decrease was noted in acute malnutrition rate overall (from $17.7 \%$ at baseline to $12.1 \%$ at $3^{\text {rd }}$ month and $6.7 \%$ at $6^{\text {th }}$ month, $\mathrm{p}<0.001$ for each) and in all subdiagnosis groups other than neuro-immune disorders (Table II).

\section{Anthropometric measurements}

Baseline values for WFH were significantly lower in malnourished than in non-malnourished patients (101.96 \pm 18.23 vs. $106.4 \pm 16.71$, $\mathrm{p}=0.034)$. When compared to baseline values, a significant improvement was noted in mean values for SD weight (increased to $-1.04 \pm 0.35$, $\mathrm{p}=0.035$ ), weight $\%$ (increased to $28.27 \pm 24.87$, $\mathrm{p}=0.037$ ), WFH (increased to $81.42 \pm 8.17$, $\mathrm{p}=0.045)$ among malnourished patients after 6-month nutritional support (Table III).

\section{NQ assessment}

In NQ-10 item assessment, at least one item was found to be positive in $66.0 \%$ of acutely malnourished patients including 58.0\%, 81.0\% and $92.0 \%$ of patients with mild, moderate and severe acute malnutrition, respectively (Table IV).

Gastrointestinal problems were the most common problems as noted in 102 (54.3\%) out of 188 acutely malnourished patients (Table IV).

Of 102 patients consulted with pediatric gastroenterology department, 30 patients were diagnosed with gastroesophageal reflux disease and received anti-reflux treatment, 19 patients were diagnosed with oropharyngeal dysfunction (6 patients managed via nasogastric tube, 4 patients with percutaneous endoscopic gastrostomy). Patients referred to psychiatry $(\mathrm{N}=14)$ received psychiatric medications and those referred to social service departments $(\mathrm{N}=5)$ received economic support to obtain enteral nutrition products.

NQ 4-item set of "red flags" revealed that prolonged meal time, meal time stressful to child or parent, lack of weight gain not just weight loss and cough during feeding were evident in $45.2 \%, 46.8 \%, 36.7 \%$ and $14.8 \%$ of patients with acute malnutrition, respectively, while being more commonly in patients with severe malnutrition (Table V).

Prolonged meal time in case of neuromuscular 


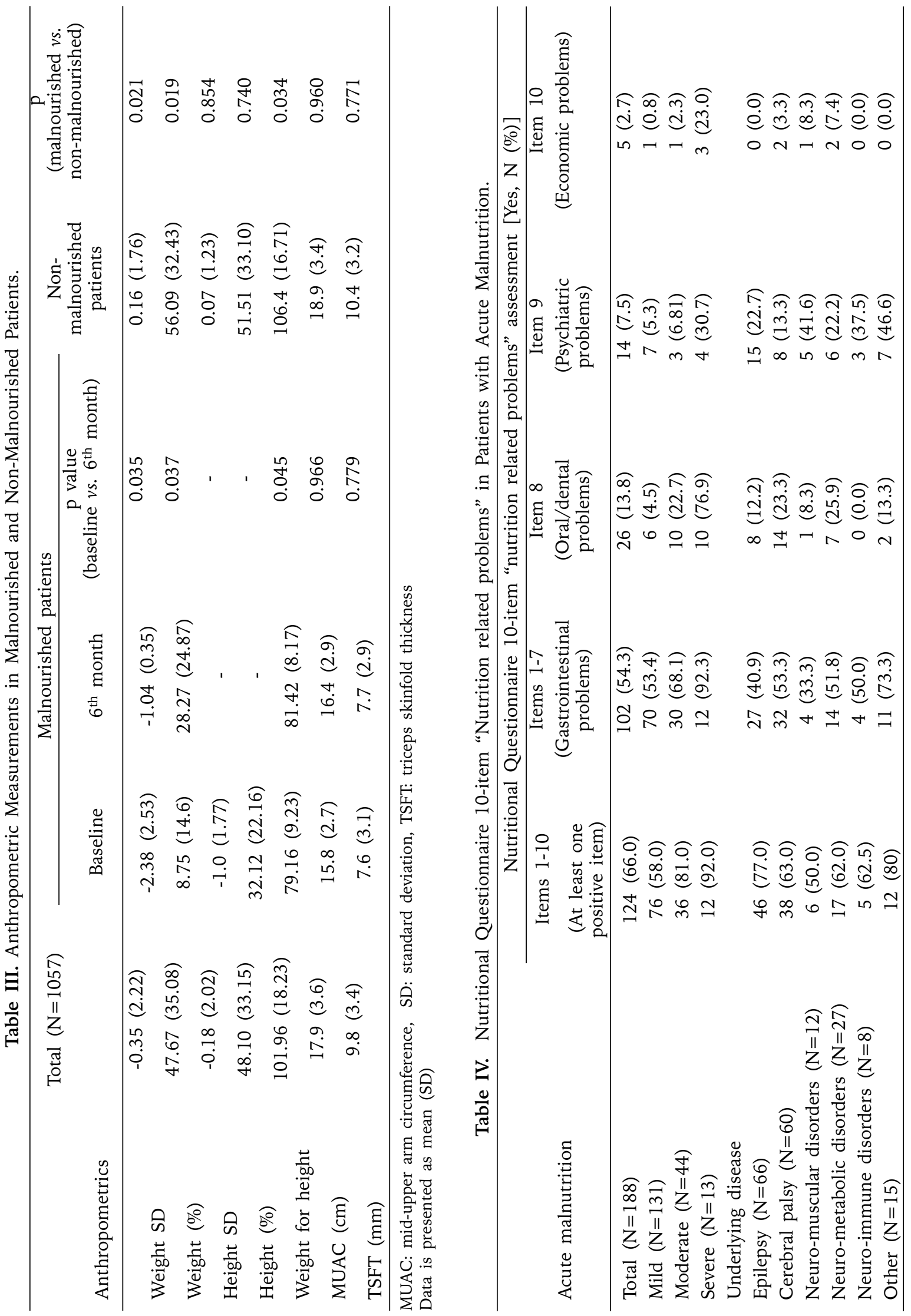


Table V. Nutritional Questionnaire 4-item Set of "Red Flags" in Patients with Acute Malnutrition.

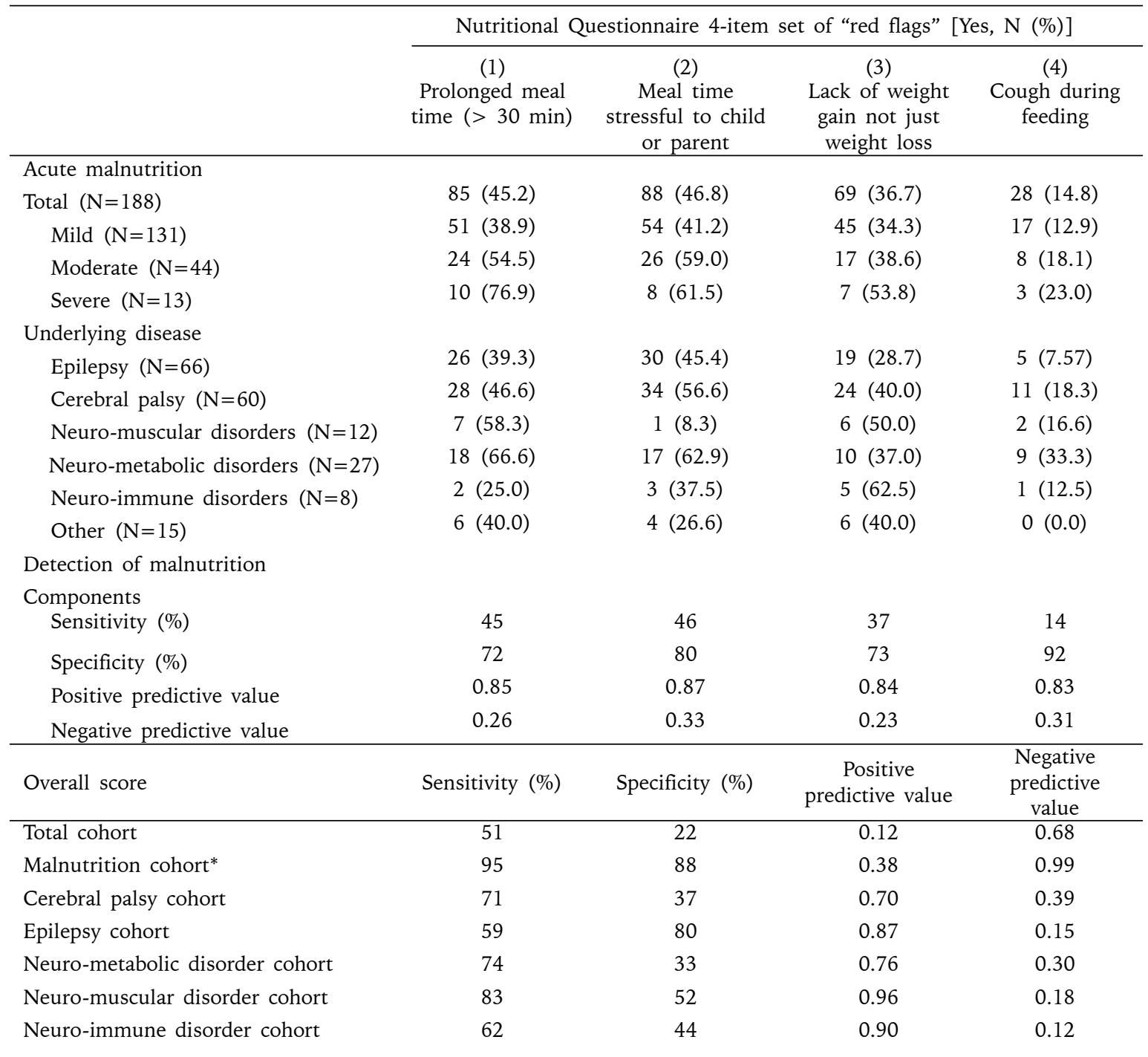

*Scored at the $6^{\text {th }}$ months of the nutritional suppor

(58.3\%) and neuro-metabolic $(66.6 \%)$ disorders, meal time stressful to child or parent in case of epilepsy (45.4\%) and CP (56.6\%) and lack of weight gain not just weight loss in case of neuro-immune disorders $(62.5 \%)$ were more common (Table V).

PPV of the questions for prediction of malnutrition ranged from 0.83 to 0.87 . Overall, use of NQ 4-item set of "red flags" was associated with high sensitivity (95\%), specificity (88\%) and NPV (0.99) in detection of malnutrition in the malnutrition cohort. However, the sensitivity, the specificity, and the predictive values of the "red flags" were not significantly high in the total cohort and in the other subgroup of the study population (Table V).

\section{Discussion}

Our findings in a cohort of pediatric neurology outpatients revealed $17.7 \%$ prevalence of acute malnutrition and higher likelihood of malnutrition in cases of CP (36.0\%). Chronic malnutrition was identified in $11.1 \%$ of the cases. Nutritional support was associated with significant decrease in percentage of malnourished patients starting from the $3^{\text {rd }}$ month of therapy along with improved 
anthropometrics. Feeding difficulties and nutritional problems were evident in a considerable portion of malnourished patients and both were more common in those with severe malnutrition.

The prevalence of malnutrition in children with neurological disabilities was reported to range from 13 to $52 \%^{11}$, while much higher rates of malnutrition were noted among hospitalized pediatric neurology patients based on WFH (42.6\%, mild in $25.9 \%$ ) and Subjective Global Nutritional Assessment (SGNA) tool (52.7\%, mild in $41.8 \%) .{ }^{15}$

In our cohort, identified based on anthropometrics using NCHS WFH centiles, malnutrition rate was evident in $17.7 \%(12.3 \%$ for mild malnutrition) of pediatric neurology outpatients. While epilepsy was the most prevalent underlying neurological disorder in our cohort, only $13.8 \%$ of patients with epilepsy were found to be malnourished. In contrast, past studies revealed much higher rates for malnutrition in patients with epilepsy ${ }^{15}, 16$ with two-fold higher rates as compared with other neurological disabilities $(62.1 \%$ vs. $37.9 \%$ according SGNA and $65.2 \% v$ s. $34.8 \%$ according to $\mathrm{WFH}) .{ }^{15}$

In our cohort, CP was the neurological diagnosis associated with highest prevalence of malnutrition rate, supporting the consideration of children with CP to be at an increased risk of malnutrition even under high-resource settings including appropriate environment and regular medical attention. ${ }^{15,16}$

According to NQ findings, at least one of 10 -item set of gastrointestinal, oral/dental, psychiatric and economic problems was found to be positive in $66.0 \%$ our patients with malnutrition. Analysis of NQ4- item set of "red flags" revealed that prolonged meal time, meal time stressful to child or parent, lack of weight gain not just weight loss and cough during feeding were evident in $45.2 \%, 46.8 \%, 36.7 \%$ and $14.8 \%$ of patients. Notably, an increase in frequency of both nutritional problems and feeding difficulties were noted with severity of malnutrition.

This seems to indicate a role of nutritional problems and feeding difficulties in both induction and deterioration of malnutrition and consistent with the wide-ranging ill effects of malnutrition on motor, neurological and psychological function. ${ }^{17}$ Likewise, nonnutritional factors such as socioeconomic problems, lack of organized services and psychological problems have been suggested to be associated with poor nutritional status in children with neurological disability. ${ }^{16,18}$

In our cohort, gastrointestinal problems were the most prevalent problems overall $(54.3 \%)$, and in all neurological sub-diagnoses groups (ranged from $40.9 \%$ to $53.3 \%$ ), as followed by oral-dental problems, particularly in case of CP $(23.3 \%)$ and neuro-metabolic disorders $(25.9 \%)$, and psychiatric problems particularly in case of epilepsy (22.7\%) and neuromuscular disorders $(41.6 \%)$. This seems notable given the frequent presence of gastrointestinal problems among children with neurodevelopmental disabilities that include oral motor dysfunction and the consequent development of feeding difficulties, risk of aspiration, prolonged feeding times, and malnutrition. ${ }^{19}$

Feeding time is considered as a reliable measure of the severity of feeding impairment in children with neurological disability ${ }^{11}$. In our cohort, prolonged meal times were evident in half of malnourished children overall and in $77 \%$ of severely malnourished children. This seems to support that longer time spent by mothers for feeding in children with disabilities as compared to non-disabled children (3.5 hour vs. 0.8 hour per day on average $)^{11,20,21}$, as well as failure of even long meal times to compensate for the severity of feeding impairment and malnutrition. ${ }^{11,21}$

Nonetheless it should be noted that relying solely on mothers' reports of mealtime durations is considered challenging with a substantial discrepancy between the lengthy durations of mealtimes reported by mothers and the far shorter mealtimes actually observed in reality. ${ }^{22}$

Accordingly, our findings revealed that while the individual questions had a quite favorable PPV (ranged from 0.83 to 0.87 ) for detecting malnutrition, overall predictive scores of the set of "red flags" were not significantly high in the total cohort which might be linked to participants' perception of the questions. Further studies are needed to validate clinical value of the set of "red flags" in screening of malnutrition in pediatric neurology patients 
from different ethnic and cultural backgrounds. Improved oral intake or appropriately selected clinical nutrition was shown to restore a normal nutritional status and correct clinical consequences of malnutrition. ${ }^{13,23,24}$ Accordingly, implementation of 6-month nutritional support in our cohort of acutely malnourished patients was associated with improved anthropometrics and decrease in rate of malnutrition. Hence, providing data on benefits of restoring normal nutritional status in malnourished children with disabilities our findings support the consensus among clinicians and researchers is that malnutrition in children with neurological disability warrants treatment. ${ }^{4}$

However, in accordance with consistently reported insufficient knowledge of nutritional support among physicians ${ }^{8}$, malnutrition is generally under-recognized and thus unmanaged in clinical practice with low percentage of high-risk patients being referred for nutritional assessment and nutritional intervention. ${ }^{10}$ NQ-4-item set of red flags had more favorable sensitivity and specificity ratios in malnutrition cohort rather than overall cohort in our study. This seems to emphasize the likely role of increased awareness about feeding/swallowing related malnutrition signs among parents of pediatric neurology patients to improve predictive potential of using NQ set of "red flags" in pediatric neurology outpatient clinics. This seems notable given that estimation of potential signs of malnutrition enables making specific instructions for feeding patterns as well as appropriate decision making for evaluation and management by physicians, nurses and/ or therapists. ${ }^{17,25}$

In this regard our findings indicate that assessment of nutritional status and global nutritional support is essential for the care of neurologically impaired children with potential benefit of assessing feeding difficulties and nutritional problems besides anthropometrics in identifying early feeding/swallowing related signs of malnutrition and making more appropriate therapeutic decisions to reveal optimal health and well-being of malnourished children.

Certain limitations to this study should be considered. First, our study was limited by issues inherent in observational methodology including the potential for not capturing, or misclassifying factors that affect clinical decision making, and thus the likelihood of main selection bias and confounding is possible. Second, albeit the overall sample size for the reliability evaluation was considered sufficient, dividing children into diagnostic sub-groups resulted in underlying neurological diagnosis groups to be relatively small, which might reach statistical significance concerning malnutrition prevalence before $v s$. after the nutritional support. Nonetheless, providing data on combined analysis of malnutrition and feeding difficulties and effectiveness of nutritional support in a wide-spectrum of pediatric neurological disabilities, our findings represent a valuable contribution to the literature.

In conclusion, our findings in a cohort of pediatric neurology outpatients revealed that $17.1 \%$ of overall patients were acutely malnourished along with higher prevalence of malnutrition in in underlying diagnosis of $\mathrm{CP}$ and higher likelihood of nutritional problems and feeding difficulties in severe malnutrition. Given the association of 6-month nutritional support with improved anthropometrics and decreased percentage of malnourished patients, our findings indicate that increased awareness of nutritional status and nutritional support is essential for the care of neurologically impaired children with potential benefit of identifying early feeding/swallowing related signs of malnutrition. Further studies are needed to validate clinical value of using the NQ set of "red flags" in screening of malnutrition and to identify optimal nutritional interventions in pediatric neurology patients from different ethnic and cultural backgrounds.

\section{REFERENCES}

1. Kerac M, Postels DG, Mallewa M, et al. The interaction of malnutrition and neurologic disability in Africa. Semin Pediatr Neurol 2014; 21: 42-49.

2. Quitadamo P, Thapar N, Staiano A, Borrelli O. Gastrointestinal and nutritional problems in neurologically impaired children. Eur J Paediatr Neurol 2016; 20: 810-815.

3. Brooks J, Day S, Shavelle R, Strauss D. Low weight, morbidity, and mortality in children with cerebral palsy: New clinical growth charts. Pediatrics 2011; 128: e299-e307.

4. Kuperminc M, Stevenson RD. Growth and nutrition disorders in children with cerebral palsy. Dev Disabil Res Rev 2008; 14: 137-146. 
5. Schwartz SM, Corredor J, Fisher-Medina J, Cohen J, Rabinowitz S. Diagnosis and treatment of feeding disorders in children with developmental disabilities. Pediatrics 2001; 108: 671-676.

6. World Health Organization (WHO). WHO child growth standards and the identification of severe acute malnutrition in infants and children: A Joint Statement by the World Health Organization and the United Nations Children's Fund 2009. Available at: http://www. who.int/nutrition/publications/severemalnutrition/ 9789241598163_eng.pdf (Accessed on Feb 2017)

7. Wong SS, Derry F, Sherrington K, Gonzales G. An audit to evaluate the use of nutrition screening tool in the National Spinal Injury Centre, Stoke Mandeville Hospital, Buckinghamshire Hospitals NHS Trust. Proc Nutr Soc 2009; 68 (OCE 1): E53.

8. Moore GE, Lindenmayer AW, McConchie GA, Ryan MM, Davidson ZE. Describing nutrition in spinal muscular atrophy: A systematic review. Neuromuscul Disord 2016; 26: 395-404.

9. Wong S, Graham A, Hirani SP, Grimble G, Forbes A. Validation of the Screening Tool for the Assessment of Malnutrition in Paediatrics (STAMP) in patients with spinal cord injuries (SCIs). Spinal Cord 2013; 51: 424-429.

10. Wong S, Graham A, Harini SP, Grimble G, Forbes A. Profile and prevalence of malnutrition in children with spinal cord injuries-assessment of the Screening Tool for Assessment of Malnutrition in Paediatrics (STAMP). Spinal Cord 2012; 50: 67-71.

11. Sullivan PB, Lambert B, Rose M, Ford-Adams M, Johnson A, Griffiths P. Prevalence and severity of feeding and nutritional problems in children with neurological impairment: Oxford Feeding Study. Dev Med Child Neurol 2000; 42: 674-680.

12. Patrick J, Boland M, Stoski D, Murray GE. Rapid correction of wasting in children with cerebral palsy. Dev Med Child Neurol 1986; 28: 734-739.

13. Sanders KD, Cox K, Cannon R, et al. Growth response to enteral feeding by children with cerebral palsy. JPEN J Parenter Enteral Nutr 1990; 14: 23-26.

14. Arvedson JC. Feeding children with cerebral palsy and swallowing difficulties. Eur J Clin Nutr 2013; 67 (Suppl 2): S9-S12.
15. Behesti M, Imanzadeh F, Shahidi N. Evaluation of the nutritional status in children admitted to the neurology ward of Mofid Children's Hospital. Iran J Child Neurology 2010; 3: 51-57.

16. Herrera-Anaya E, Angarita-Fonseca A, HerreraGalindo VM, Martínez-Marín RD, Rodríguez-Bayona $\mathrm{CN}$. Association between gross motor function and nutritional status in children with cerebral palsy: A cross-sectional study from Colombia. Dev Med Child Neurol 2016; 58: 936-941.

17. Palisano R, Rosenbaum P, Walter S, Russell D, Wood E, Galuppi B. Development and reliability of a system to classify gross motor function in children with cerebral palsy. Dev Med Child Neurol 1997; 39: 214-223.

18. Jauhari P, Bhargava R, Bhave A, Kumar C, Kumar R. Comorbidities associated with intellectual disability among pediatric outpatients seen at a teaching hospital in Northern India. J Policy Pract Intellect Disabil 2012; 9: 10-16.

19. Sullivan PB. Gastrointestinal disorders in children with neurodevelopmental disabilities. Dev Disabil Res Rev 2008; 14: 128-136.

20. Johnson CB, Deitz JC. Time use of mothers with preschool children: A pilot study. Am J Occup Ther 1985; 39: 578-583.

21. Gisel EG, Patrick J. Identification of children with cerebral palsy unable to maintain a normal nutritional state. Lancet 1988; 1: 283-286.

22. Reilly S. Prevalence and severity of feeding and nutritional problems in children with neurological impairment: Oxford Feeding Study. Dev Med Child Neurol 2001; 43: 358.

23. Soylu OB, Unalp A, Uran N, et al. Effect of nutritional support in children with spastic quadriplegia. Pediatr Neurol 2008; 39: 330-334.

24. Crepin S, Godet B, Chassain B, Preux PM, Desport JC. Malnutrition and epilepsy: A two-way relationship. Clin Nutr 2009; 28: 219-225.

25. Garg BP. Dysphagia in children: An overview. Semin Pediatr Neurol 2003; 10: 252-254. 